\title{
Liter per Minute per Milligram per Meter Squared
}

National Cancer Institute

\section{Source}

National Cancer Institute. Liter per Minute per Milligram per Meter Squared. NCI

Thesaurus. Code C120754.

A unit of concentration equal to liter per minute divided by milligram per meter squared. 